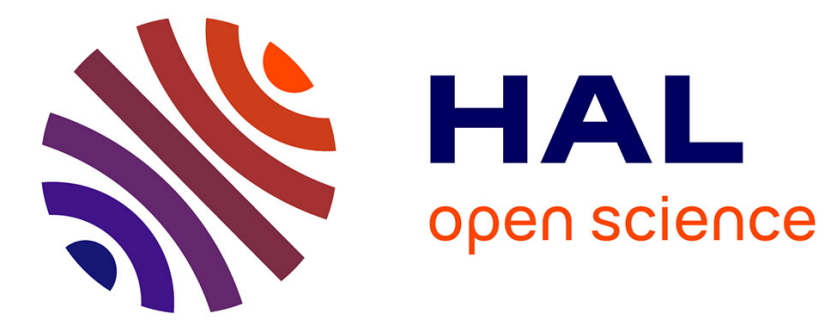

\title{
Drift-Alfvén vortices at the ion Larmor radius scale
}

O. G. Onishchenko, Vladimir Krasnoselskikh, O. A. Pokhotelov

\section{To cite this version:}

O. G. Onishchenko, Vladimir Krasnoselskikh, O. A. Pokhotelov. Drift-Alfvén vortices at the ion Larmor radius scale. Physics of Plasmas, 2008, 15 (2), pp.022903. 10.1063/1.2844744 . insu-02515691

\section{HAL Id: insu-02515691 \\ https://hal-insu.archives-ouvertes.fr/insu-02515691}

Submitted on 23 Apr 2021

HAL is a multi-disciplinary open access archive for the deposit and dissemination of scientific research documents, whether they are published or not. The documents may come from teaching and research institutions in France or abroad, or from public or private research centers.
L'archive ouverte pluridisciplinaire $\mathbf{H A L}$, est destinée au dépôt et à la diffusion de documents scientifiques de niveau recherche, publiés ou non, émanant des établissements d'enseignement et de recherche français ou étrangers, des laboratoires publics ou privés. 


\section{Drift-Alfvén vortices at the ion Larmor radius scale}

Cite as: Phys. Plasmas 15, 022903 (2008); https://doi.org/10.1063/1.2844744

Submitted: 01 October 2007 . Accepted: 24 January 2008 . Published Online: 28 February 2008

O. G. Onishchenko, V. V. Krasnoselskikh, and O. A. Pokhotelov

\section{ARTICLES YOU MAY BE INTERESTED IN}

Large-scale Alfvén vortices

Physics of Plasmas 22, 122901 (2015); https://doi.org/10.1063/1.4936978

Pseudo-three-dimensional turbulence in magnetized nonuniform plasma

The Physics of Fluids 21, 87 (1978); https://doi.org/10.1063/1.862083

Current-vortex filament model of nonlinear Alfvén perturbations in a finite-pressure plasma

Physics of Plasmas 5, 3833 (1998); https://doi.org/10.1063/1.873103 


\title{
Drift-Alfvén vortices at the ion Larmor radius scale
}

\author{
O. G. Onishchenko, ${ }^{1, a)}$ V. V. Krasnoselskikh, ${ }^{1, b)}$ and O. A. Pokhotelov ${ }^{2, c)}$ \\ ${ }_{1}^{1}$ LPCE/CNRS, 3A, Avenue de la Recherche Scientifique, 45071 Orléans Cedex 2, France \\ ${ }^{2}$ Department of Automatic Control and Systems Engineering, University of Sheffield, \\ Sheffield S13JD, United Kingdom
}

(Received 1 October 2007; accepted 24 January 2008; published online 28 February 2008)

\begin{abstract}
The theory of nonlinear drift-Alfvén waves with the spatial scales comparable to the ion Larmor radius is developed. It is shown that the set of equations describing the nonlinear dynamics of drift-Alfvén waves in a quasistationary regime admits a solution in the form of a solitary dipole vortex. The vortex structures propagating perpendicular to the ambient magnetic field faster than the diamagnetic ion drift velocity possess spatial scales larger than the ion Larmor radius, and vice versa. The variation of the vortex impedance and spatial scale as the function of the vortex velocity is analyzed. It is shown that incorporation of the finite electron temperature effects results in the appearance of a minimum in the dependence of the vortex impedance on the vortex velocity. This leads to the existence of the vortex structures with the smallest impedance. These structures are probably the most favorable energetically and can easily be excited in space plasmas. The relevance of theoretical results obtained to the Cluster observations in the magnetospheric cusp and magnetosheath is stressed. (C) 2008 American Institute of Physics. [DOI: 10.1063/1.2844744]
\end{abstract}

\section{INTRODUCTION}

The drift-Alfvén waves, whose perpendicular wavelengths often determine the fine structure of many auroral processes, play an important role in the electrodynamic coupling between the ionosphere and magnetosphere. They substantially contribute to the formation of the discrete fluxes of low-energy electrons and suprathermal ions. Occasionally these waves have been observed in the highly nonlinear regime, e.g., in the form of a two-dimensional solitary vortex or vortex street, in which the perturbed electric and magnetic fields exhibit regular rotation together with the particles trapped inside the structure. Two decades ago the spatial structures of that form were registered on board the Intercosmos-Bulgaria-1300 (ICB-1300) satellite in the auroral ionosphere. ${ }^{1}$ Unfortunately, the single satellite measurements available during that time do not allow the separation of spatial variations from temporal. Recently, interest in the drift-Alfvén vortex structures has been considerably reinforced by the possibility of multisatellite wave measurements during the four Cluster spacecraft mission. Contrary to ICB1300 measurements, this new experiment provides a unique opportunity to detect the coherent vortex structures in situ by separating the temporal and spatial perturbations. ${ }^{2,3}$ It has been found that vortex structures have been observed occasionally both in the magnetospheric cusp and in the Earth's magnetosheath. ${ }^{2-4}$ The observed waves were of an electromagnetic nature and their impedance was of the order of the local Alfvén velocity. The structures possess rather small spatial scales of the order of the ion Larmor radius. ${ }^{2,3}$ The perpendicular vortex velocity was substantially smaller than the local Alfvén speed. They have been identified as vortices

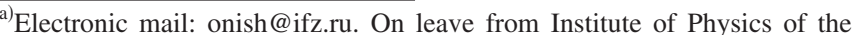
Earth, 123995 Moscow, Russia.

b) Electronic mail: vkrasnos@cnrs0orleans.fr.

${ }^{c}$ Electronic mail: o.a.pokhotelov@sheffield.ac.uk.
}

of the drift-Alfvén waves. The existence of the vortex structures points out on the intermittent nonlocal turbulence of the Alfvén and drift-Alfvén wave interaction with nonlocal fluxes of the wave energy and generalized enstrophy in the inertial region. ${ }^{5}$

The previous theories ${ }^{6-14}$ of nonlinear vortex structures in a magnetized collisionless plasma were restricted to consideration of the relatively long wavelength limit when the perpendicular spatial scale $L$ is larger than the ion Larmor radius $\rho_{i}$, i.e., $L / \rho_{i} \gg 1$. The aim of the present paper is to investigate the nonlinear dynamics of drift-Alfvén waves and reveal at which conditions the vortex structures with spatial scales comparable to the ion Larmor radius can exist in the near Earth environment.

The paper is organized as follows. In Sec. II, the general dispersion relation for the drift-Alfvén waves is discussed. Two-fluid hydrodynamic equations describing the nonlinear dynamics of small-scale drift-Alfvén waves are obtained in Sec. III. The quasistationary solutions of the nonlinear equations are found and analyzed in Sec. IV. It is shown that they admit a solution in the form of a solitary vortex with spatial scales comparable to the ion Larmor radius. The typical velocities and impedance of such vortex structures are investigated. Our discussion and conclusions are found in Sec. V.

\section{DRIFT-ALFVÉN-WAVE DISPERSION RELATION}

Let us consider an inhomogeneous low- $\beta$ plasma immersed in a uniform external magnetic field $\mathbf{B}_{0}$. We use a local Cartesian coordinate system whose $z$ axis is parallel to $\mathbf{B}_{0}$, the $x$ axis is along the plasma density gradient, and the $y$ axis completes the triad. The linear dispersion relation for the drift-Alfvén waves in such a plasma was discussed in Refs. 15-17. The linear dispersion relation of the drift-Alfvén waves with arbitrary wavelength $\lambda_{\perp}$ compared to the ion Larmor radius is ${ }^{16}$ 


$$
\begin{aligned}
\omega(\omega- & \left.\omega_{e *}\right)\left(\omega-\omega_{i *}\right)\left[1-e^{-z_{i}} I_{0}\left(z_{i}\right)\right] \\
= & k_{z}^{2} v_{A}^{2} z_{i}\left[\omega\left[1+\left(T_{e} / T_{i}\right)\left(1-e^{-z_{i}} I_{0}\left(z_{i}\right)\right)\right]\right. \\
& \left.-\omega_{e *} e^{-z_{i}} I_{0}\left(z_{i}\right)\right],
\end{aligned}
$$

where $\omega$ is the wave frequency, $\omega_{i *}=k_{y} T_{i} \kappa_{n} / e B_{0}$ and $\omega_{e *}=-k_{y} T_{e} \kappa_{n} / e B_{0}$ are the ion and electron drift frequencies, respectively, $T_{i(e)}$ is the ion (electron) temperature, $k_{\perp}^{2}=k_{x}^{2}$ $+k_{y}^{2}, k_{x}, k_{y}$, and $k_{z}$ are the $x, y$, and $z$ components of the wave vector $\mathbf{k}$, respectively, $v_{A}=B_{0} /\left(\mu_{0} n_{0} m_{i}\right)^{1 / 2}$ is the Alfvén velocity, $\mu_{0}$ is the permeability of free space, $m_{i}$ is the ion mass, $\kappa_{n}=d \ln \left(n_{0}\right) / d x, z_{i}=k_{\perp}^{2} \rho_{i}^{2}, \rho_{i}=\left(T_{i} / m_{i}\right)^{1 / 2} / \omega_{c i}$ is the ion Larmor radius, $\omega_{c i}=e B_{0} / m_{i}$ is the ion gyrofrequency, $e$ is the magnitude of the electron charge, and $I_{0}$ is the modified Bessel function of the first kind.

In the small ion Larmor radius approximation, $z_{i}=k_{\perp}^{2} \rho_{i}^{2}$ $\ll 1$, one can make use of an expansion $1-e^{-z_{i}} I_{0}\left(z_{i}\right) \simeq z_{i}[1$ $\left.-(3 / 4) z_{i}\right]$. Then the dispersion relation (1) reduces to

$$
\omega^{2}\left(1-k_{\perp}^{2} \rho_{i}^{2} \Lambda\right)-\omega \omega_{i *}=k_{z}^{2} v_{A}^{2},
$$

where

$$
\Lambda=\frac{3}{4}\left(1-\frac{\omega_{i *}}{\omega}\right)+\frac{T_{e}}{T_{i}} \frac{k_{z}^{2} v_{A}^{2}}{\omega^{2}}\left(1-\frac{\omega_{e *}}{\omega}\right)^{-1} .
$$

The nonlinear drift-Alfvén waves, which in the linear approximation are described by the dispersion relation (2), were studied extensively in Refs. 10 and 11. It was shown that they can exist in the form of the dipole vortex with the perpendicular spatial scales larger than the ion Larmor radius, i.e., $L / \rho_{i} \gg 1$.

When plasma inhomogeneity is neglected, $\omega_{i *} \rightarrow 0$ and $\omega_{e *} \rightarrow 0$, Eq. (2) recovers the ordinary dispersion relation for the kinetic Alfvén waves (KAWs), ${ }^{18}$

$$
\omega^{2}=k_{z}^{2} v_{A}^{2}\left[1+k_{\perp}^{2} \rho_{s}^{2}\left(1+3 T_{i} / 4 T_{e}\right)\right],
$$

where $\rho_{s}=c_{s} / \omega_{c i}$ and $c_{s}=\left(T_{e} / m_{i}\right)^{1 / 2}$ are the ion acoustic gyroradius and the ion acoustic speed, respectively. The dispersion relation (2) can be regarded as a variety of the well known dispersion relation for the KAWs modified by the presence of the plasma inhomogeneity.

In the case of most importance when $z_{i}$ takes the finite values of the order of unity or larger (corresponding to Cluster observations), one can employ the so-called Padé approximation $1-e^{-z_{i}} I_{0}\left(z_{i}\right) \simeq z_{i} /\left(1+z_{i}\right)$. It has been shown $^{18,19}$ that such an approximation of the term $1-e^{-z_{i}} I_{0}\left(z_{i}\right)$ is suitable for the entire range of $z_{i}$. It is almost as accurate as the approximation used in Ref. 20 when $z_{i}<1$. With the use of this relation, the dispersion relation (1) reduces to the form that allows us to incorporate the full ion Larmor radius effects,

$$
\omega\left(\omega-\omega_{* i}\right)=k_{z}^{2} v_{A}^{2}\left[1+k_{\perp}^{2} \rho_{i}^{2}\left(1+T_{e} / T_{i}\right)\left(1-\omega_{* e} / \omega\right)^{-1}\right] .
$$

In what follows, we will derive a set of nonlinear equations describing the dynamics of the drift-Alfvén waves that in the linear approximation are described by dispersion relation (5).

\section{NONLINEAR EQUATIONS}

To study the nonlinear dynamics of drift-Alfvén waves, we make use of the two-potential representation, $E_{z} \equiv \mathbf{E} \cdot \hat{\mathbf{z}}$ $=-\partial_{t} A-\partial_{z} \phi, \mathbf{E}_{\perp}=-\nabla_{\perp} \phi$, and $\mathbf{B}_{\perp}=\nabla A \times \hat{\mathbf{z}}$, where $\mathbf{E}$ and $\mathbf{B}$ are perturbations of the electric and magnetic fields, respectively, $\hat{\mathbf{z}}$ is the unit vector along the ambient magnetic field $\mathbf{B}_{0}, \partial_{t} \equiv \partial / \partial t$ and $\partial_{z} \equiv \partial / \partial z$, and the subscripts $z$ and $\perp$ denote the components along and perpendicular to $\hat{\mathbf{z}}$, respectively. Furthermore, $\phi$ is the scalar potential of the electric field and $A$ is the $z$ component of the vector potential. Since we consider a low- $\beta$ plasma $\left(1 \gg \beta \gg m_{e} / m_{i}\right)$, the compressional component of the magnetic field can be disregarded.

In the low-frequency approximation, we decompose the electron velocity $\mathbf{v}_{e}$ as $\mathbf{v}_{e}=\mathbf{v}_{E}+\mathbf{v}_{e D}+v_{z e}\left(\hat{\mathbf{z}}+\mathbf{B}_{\perp} / B_{0}\right)$, where $\mathbf{v}_{E}$ is the $\mathbf{E} \times \mathbf{B}$ velocity, $\mathbf{v}_{e D}=-T_{e}\left(m_{i} n_{0} \omega_{c i}\right)^{-1}\left(\hat{\mathbf{z}} \times \nabla_{\perp} n_{0}\right)$ is the electron diamagnetic drift velocity, and $v_{z e}$ is the parallel electron speed. The $z$ component of the electric current can be found from the Ampére law and is $j_{z}=-\mu_{0}^{-1} \nabla_{\perp}^{2} A$. We assume that the ion velocity parallel to the magnetic field is small and thus the parallel electric current $j_{z}$ is driven only by the electrons, i.e., $j_{z}=-e n_{0} v_{z e}$ and $v_{z e}=\nabla_{\perp}^{2} A / \mu_{0} e n_{0}$. Taking into account that $\nabla \cdot \mathbf{v}_{E}=0$ and $\nabla \cdot\left(n_{e} \mathbf{v}_{e D}\right)=0$, the electron continuity equation, $\partial_{t} n_{e}+\nabla_{\perp} \cdot\left(n_{e} \mathbf{v}_{e}\right)+n_{0} d_{z} v_{z e}=0$, reduces to

$$
n_{0}^{-1} d_{t}^{0} \widetilde{n}_{e}+v_{e D} \partial_{y} \Phi_{e}+v_{A}^{2} \rho_{s}^{2} d_{z} \nabla_{\perp}^{2} A_{e}=0 .
$$

Here $n_{e}=n_{0}+\widetilde{n}_{e}, \widetilde{n}_{e}$ is the perturbed electron number density, $\Phi_{e} \equiv e \phi / T_{e}, A_{e} \equiv e A / T_{e}, d_{t}^{0} \equiv \partial_{t}+\mathbf{v}_{E} \cdot \nabla, d_{z} \equiv \partial_{z}+B_{0}^{-1} \mathbf{B}_{\perp} \cdot \nabla$, $\partial_{t} \equiv \partial / \partial t$, and $\partial_{y} \equiv \partial / \partial y$.

The equation for the electron momentum balance along the total magnetic field $\mathbf{B}_{0}+\mathbf{B}_{\perp}$ is $e n_{0} E_{\|}+d_{z} \widetilde{p}_{e}=0$, where $E_{\|}=E_{z}+B_{0}^{-1} \mathbf{B}_{\perp} \cdot \mathbf{E}_{\perp}$ is the electric field component along the total magnetic field and $\widetilde{p}_{e}=T_{e} \widetilde{n}_{e}$ is the electron pressure perturbation. We note that the term due to the electron inertia is neglected since $\beta \gg m_{e} / m_{i}$. Then we have

$$
\left(\partial_{t}+v_{e D} \partial_{y}\right) A_{e}+d_{z}\left(\Phi_{e}-\tilde{n}_{e} / n_{0}\right)=0 .
$$

In order to close the system of nonlinear equations (6) and (7), it is necessary to supplement it by the equation for the ion motion. It can be considered as two-dimensional, $\mathbf{v}_{i}=\mathbf{v}_{\perp i}$. To calculate the ion velocity, we make use of the ion momentum equation

$$
\frac{e}{m_{i}}\left(\mathbf{v}_{i} \times \mathbf{B}+\mathbf{E}\right)-\frac{\nabla_{\perp} p_{i}}{m_{i} n_{i}}=d_{t} \mathbf{v}_{i},
$$

where $p_{i}=n_{i} T_{i}$ is the ion pressure and $d_{t} \equiv \partial_{t}+\mathbf{v}_{i} \cdot \nabla$ is the Lagrangian derivative.

A power series expansion of Eq. (8) on small parameter $\varepsilon=\omega_{c i}^{-1} d_{t} \ll 1$ yields

$$
\mathbf{v}_{i} \simeq \mathbf{v}_{E}+\mathbf{v}_{i D}+\omega_{c i}^{-1}\left[\hat{\mathbf{z}} \times\left(\partial_{t}+\left(\mathbf{v}_{E}+\mathbf{v}_{i D}\right) \cdot \nabla\right)\left(\mathbf{v}_{E}+\mathbf{v}_{i D}\right)\right] .
$$

Here $v_{i D}=\left(m_{i} n_{i} \omega_{c i}\right)^{-1}\left(\hat{\mathbf{z}} \times \nabla_{\perp} p_{i}\right)$ is the ion diamagnetic drift velocity. The last (polarization) term on the right-hand side of Eq. (9) can be decomposed as $\omega_{c i}^{-1}\left[\hat{\mathbf{z}} \times\left(\partial_{t}+\left(\mathbf{v}_{E}+\mathbf{v}_{i D}\right) \cdot \nabla\right)\right.$ $\left.\times\left(\mathbf{v}_{E}+\mathbf{v}_{i D}\right)\right] \equiv \mathbf{v}_{E}^{P}+\mathbf{v}_{i D}^{P}$, where $\mathbf{v}_{E}^{P}$ and $\mathbf{v}_{i D}^{P}$ stand for the polar- 
ization parts of the ion velocity connected to the drift velocities $\mathbf{v}_{E}$ and $\mathbf{v}_{i D}$ through the relations

$$
\mathbf{v}_{E}^{P}=\frac{1}{\omega_{c i}}\left(\hat{\mathbf{z}} \times d_{t}^{0} \mathbf{v}_{E}\right)=-\rho_{i}^{2} d_{t}^{0} \nabla_{\perp} \Phi_{i}
$$

and

$$
\begin{aligned}
\mathbf{v}_{i D}^{P} & =\frac{1}{\omega_{c i}}\left(\hat{\mathbf{z}} \times\left(d_{t}^{0} \mathbf{v}_{i D}+\mathbf{v}_{i D} \cdot \nabla\left(\mathbf{v}_{E}+\mathbf{v}_{i D}\right)\right)\right) \\
& =-\frac{\rho_{i}^{2}}{n_{0}} d_{t}^{0} \nabla_{\perp} n_{i}-\frac{\rho_{i}^{3} v_{T i}}{n_{0}}\left\{n_{i}, \nabla_{\perp} \Phi_{i}\right\} .
\end{aligned}
$$

Here $\quad v_{T i}=\left(T_{i} / m_{i}\right)^{1 / 2}, \quad \Phi_{i}=e \phi / T_{i}, \quad d_{t}^{0}=\partial_{t}+\mathbf{v}_{E} \cdot \nabla=\partial_{t}$ $+\rho_{i} v_{T i}\left\{\Phi_{i}, \ldots\right\}$, and $\{A, B\} \equiv\left(\partial_{x} A\right) \partial_{y} B-\left(\partial_{y} A\right) \partial_{x} B$ denotes the Poisson bracket. With the help of Eqs. (10) and (11), the ion continuity equation $\partial_{t} n_{i}+\nabla_{\perp} \cdot\left(n_{i} \mathbf{v}_{i}\right)=0$ reduces to ${ }^{21}$

$$
d_{t}^{0} n_{i}+n_{i} \nabla \cdot\left(\mathbf{v}_{E}^{P}+\mathbf{v}_{i D}^{P}\right)=0,
$$

where the terms $\mathbf{v}_{i D} \cdot \nabla \mathbf{v}_{i D}$ have been neglected in the considered WKB (Wentzel-Kramers-Brillouin) approximation as small of the order of $\lambda_{\perp} / L$, where $L=\kappa_{n}^{-1}$ is the spatial scale of the plasma inhomogeneity. Decomposing $n_{i}=n_{0}+\widetilde{n}_{i}$, where $\widetilde{n}_{i}\left(\ll n_{0}\right)$ is the wave perturbations of the ion number density, and accounting for the polarization parts of the ion velocity Eqs. (10) and (11), from Eq. (12) one finds that

$$
\begin{aligned}
& d_{t}^{0}\left(1-\rho_{i}^{2} \nabla_{\perp}^{2}\right) \widetilde{n}_{i}-u_{* i} n_{0} \partial_{y} \Phi_{i}-n_{0} \rho_{i}^{2} d_{t}^{0} \nabla_{\perp}^{2} \Phi_{i} \\
& \quad=\rho_{i}^{3} v_{T i}\left\{\nabla_{\perp} \widetilde{n}_{i}, \nabla_{\perp} \Phi_{i}\right\} .
\end{aligned}
$$

Here the ion temperature perturbations have been neglected as small corrections of the higher order. Equations (6), (7), and (13) together with the charge neutrality condition, $\widetilde{n}_{i}=\widetilde{n}_{e}$, constitute a closed set of equations describing the nonlinear dynamics of drift-Alfvén waves in a plasma with nonzero ion temperature.

In the linear approximation, a Fourier transform of Eq. (13) gives

$$
\frac{\tilde{n}_{i}}{n_{0}}=-\left[\frac{\omega_{* i}}{\omega}+\frac{z_{i}}{1+z_{i}}\left(1-\frac{\omega_{* i}}{\omega}\right)\right] \Phi_{i} .
$$

Let us compare relation (14) with that of the fully kinetic treatment ${ }^{15}$

$$
\frac{\tilde{n}_{i}}{n_{0}}=-\left\{\frac{\omega_{* i}}{\omega}+\left[1-e^{-z_{i}} I_{0}\left(z_{i}\right)\right]\left(1-\frac{\omega_{* i}}{\omega}\right)\right\} \Phi_{i} .
$$

One sees that in the Padé approximation, relation (15) coincides with Eq. (14). This confirms that Eq. (14) adequately describes the ion density perturbations. In the linear approximation, Eqs. (6), (7), and (13) yield the local dispersion relation (5). This justifies the relevance of our hydrodynamic approach to the exact kinetic theory.

\section{VORTEX SOLUTION}

Now let us analyze the quasistationary solutions. We assume that all perturbed values depend solely on $x$ and $\eta=y$ $-u t+\alpha z$, where $u$ is the translation speed of the wave along the $y$ axis and $\alpha$ is the angle between the wave front normal and the $(x, y)$ plane. In the stationary $(x, \eta)$ frame, Eqs. (6) and (7) take the form

$$
\hat{D}_{\phi}\left(\frac{\tilde{n}_{e}}{n_{0}}-\frac{v_{e D}}{u} \Phi_{e}\right)=\frac{\alpha v_{A}^{2} \rho_{s}^{2}}{u} \hat{D}_{A} \nabla_{\perp}^{2} A_{e}
$$

and

$$
\hat{D}_{A}\left[-\frac{u}{\alpha}\left(1-\frac{v_{e D}}{u}\right) A_{e}+\Phi_{e}-\frac{\tilde{n}_{e}}{n_{0}}\right]=0,
$$

where $\hat{D}_{\phi}=\partial_{\eta}-\left(\rho_{s} c_{s} / u\right)\left(\nabla \Phi_{e} \times \nabla\right)_{z}$ and $\hat{D}_{A}=\partial_{\eta}-\left(\rho_{s} c_{s} / \alpha\right)$ $\times\left(\nabla A_{e} \times \nabla\right)_{z}$, respectively. A particular solution of Eq. (17) is

$$
\frac{\tilde{n}_{e}}{n_{0}}=\Phi_{e}-\frac{u}{\alpha}\left(1-\frac{v_{e D}}{u}\right) A_{e} .
$$

Substituting Eq. (18) into Eq. (17), one obtains

$$
\hat{D}_{\phi}\left[\left(1-\frac{v_{e D}}{u}\right)\left(\Phi_{e}-\frac{u}{\alpha} A_{e}\right)\right]=\frac{\alpha v_{A}^{2} \rho_{s}^{2}}{u} \hat{D}_{A} \nabla_{\perp}^{2} A_{e} .
$$

With the help of the equality

$$
\hat{D}_{A}\left(\Phi_{e}-\frac{u}{\alpha} A_{e}\right)=\hat{D}_{\phi}\left(\Phi_{e}-\frac{u}{\alpha} A_{e}\right),
$$

Eq. (19) reduces to

$$
\hat{D}_{A}\left[\left(1-\frac{v_{e D}}{u}\right)\left(\Phi_{e}-\frac{u}{\alpha} A_{e}\right)-\frac{\alpha v_{A}^{2}}{u} \rho_{s}^{2} \nabla_{\perp}^{2} A_{e}\right]=0 .
$$

A particular solution of Eq. (21) is

$$
\Phi_{e}=\frac{u}{\alpha}\left[1+\frac{\alpha^{2} v_{A}^{2}}{u^{2}}\left(1-\frac{v_{e D}}{u}\right)^{-1} \rho_{s}^{2} \nabla_{\perp}^{2}\right] A_{e} .
$$

With the help of relations (18) and (22) and the condition of the electroneutrality perturbations $\widetilde{n}_{e}=\widetilde{n}_{i}=\widetilde{n}$, Eq. (13) reduces to

$$
\begin{aligned}
\hat{D}_{\phi}\{[ & {\left[u\left(v_{i D}-u\right)-\alpha^{2} v_{A}^{2}\right] \nabla_{\perp}^{2}+\alpha^{2} v_{A}^{2} \rho_{i}^{2}\left(1+T_{e} / T_{i}\right) } \\
& \left.\times\left(1-v_{e D} / u\right)^{-1} \nabla_{\perp}^{4}\right\} \Phi_{i}=0 .
\end{aligned}
$$

A particular solution of Eq. (23) is

$$
\kappa^{-2} \nabla_{\perp}^{4} \Phi_{i}-\nabla_{\perp}^{2} \Phi_{i}+C\left(\Phi_{i}-u B_{0} x\right)=0,
$$

where

$$
\kappa^{2}=-\frac{\left(u^{2}-u v_{i D}-\alpha^{2} v_{A}^{2}\right)\left(1-v_{e D} / u\right)}{\alpha^{2} v_{A}^{2} \rho_{i}^{2}\left(1+T_{e} / T_{i}\right)},
$$

and $C$ is the constant value. Equation (24) is of the fourth order instead of the second order in the case of the CharneyHasegawa-Mima equation (cf. Refs. 6, 8, and 10 and references therein). Such an equation and its solution were investigated in Refs. 11-13.

Now we introduce the polar coordinates $r \equiv\left(x^{2}+\eta^{2}\right)^{1 / 2}$ and $\theta \equiv \tan ^{-1}(\eta / x)$ and then seek the solution of Eq. (24) in the dipolar form $\Phi_{i}(r, \theta)=\Phi(r) \cos \theta$. We suppose that at a certain point $r=a$, the solution of Eq. (24) is singular, so when $r>a$ (in the vortex exterior), the constant value $C$ 
vanishes and $C \neq 0$ when $r<a$ (in the vortex interior) and consider that $\Phi(a)=u B_{0} a / c$. In the external region, the solution is

$$
\Phi(r)=\Phi(a)\left[c_{1} \frac{K_{1}(\beta r / a)}{K_{1}(\beta)}+c_{2} \frac{a}{r}\right]
$$

and in the internal region

$$
\Phi(r)=\Phi(a)\left[\frac{r}{a}+c_{3} \frac{J_{1}(\gamma r / a)}{J_{1}(\gamma)}+c_{4} \frac{I_{1}(\nu r / a)}{I_{1}(\nu)}\right],
$$

where $\beta=a \kappa, c_{1}, c_{2}, c_{3}$, and $c_{4}$ are constants, and $J_{1}, I_{1}$, and $K_{1}$ are the Bessel function and the modified Bessel functions of the first and second kind, respectively. The coefficients $\gamma$ and $\nu$ are

$$
\gamma=a^{2}\left[\left(\kappa^{4}+4 \delta\right)^{1 / 2}-\kappa^{2}\right]
$$

and

$$
\nu^{2}=a^{2}\left[\left(\kappa^{4}+4 \delta\right)^{1 / 2}+\kappa^{2}\right],
$$

where $\delta=-C \kappa^{2}$. The coefficients $c_{1}, c_{2}, c_{3}$, and $c_{4}$ can be obtained from continuity of $\Phi(r), d \Phi(r) / d r, d^{2} \Phi(r) / d r^{2}$, and $d^{3} \Phi(r) / d r^{3}$ at $r=a$. The additional relation connecting the vortex parameters $\kappa, \gamma$, and $\nu$, usually called the matching condition, is obtained from a continuity of $d^{4} \Phi(r) / d r^{4}$ at $r=a$ (e.g., Refs. 11-13 for further details). It should be noted that $c_{1} \gg c_{2}$. Using this fact from Eq. (26), one concludes that in the nearby external region where the first term on the right is dominant, $\Phi(r) \propto K_{1}(\kappa r)$, whereas $\Phi(r)$ scales as $\propto r^{-1}$ at larger $r$.

We note that Eq. (25) can be obtained from the linear dispersion relation (5) with the use of substitutions $\omega / k_{y}$ $\rightarrow u$ and $k_{z} / k_{y} \rightarrow \alpha$ and taking into account that in the external region for large $r$ the asymptotics of $\Phi(r)$ scales as $\propto \exp (-\kappa r)$ so that $k_{\perp}^{2} \rightarrow-\kappa^{2}$. Therefore, Eq. (25) can be considered as a modified dispersion relation for the drift-Alfvén vortices. Vortices propagating with velocity $u<0$ or $u>0$ correspond to the electron-drift or the ion-drift modes, respectively. Below, we will restrict our consideration to driftAlfvén vortices related to the ion-drift and kinetic Alfvén modes. The impedance $E_{\perp} / B_{\perp}$ of these waves is of the order of $v_{A}$, whereas the impedance of the electron-drift waves substantially exceeds $v_{A}$. The multiplier $1-v_{e D} / u=1$ $+\left(T_{e} / T_{i}\right) v_{i D} / u$ in the denominator of Eq. (25) accounts for the electron contribution. When the vortex velocity becomes close to the electron diamagnetic drift velocity $u \simeq\left|v_{e D}\right|$, the ion- and electron-drift modes are coupled and thus this term can play an important role. From Eq. (25) one sees that localized drift Alfvén vortices exist, $\kappa^{2}>0$, i.e.,

$$
u^{2}-u v_{i D}-\alpha^{2} v_{A}^{2}<0
$$

or in the range of the vortex velocities $0 \leqslant u \leqslant u_{\max }$ with the maximum vortex velocity given by $u_{\max }=v_{i D}[1+(1$ $\left.\left.+4 \mu_{*}\right)^{1 / 2}\right] / 2$, where $\mu_{*} \equiv\left(\alpha v_{A} / v_{i D}\right)^{2}$. From Eq. (25) one can

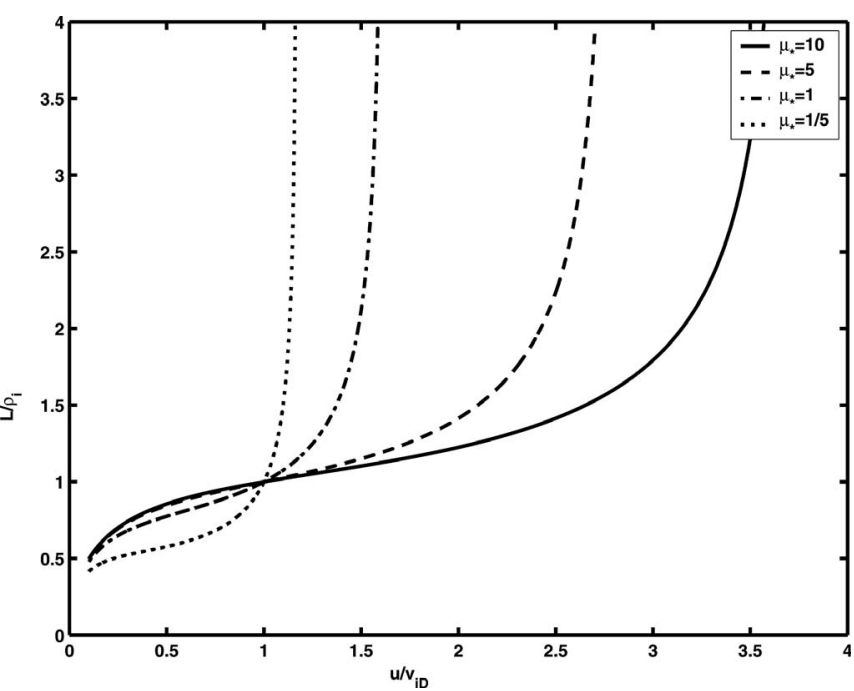

FIG. 1. A plot of the normalized vortex spatial scale as a function of normalized vortex velocity. The electron to ion temperature ratio is $T_{e} / T_{i}=0.5$. Solid, dashed, dashed-dotted, and dotted lines correspond to $\mu_{*}$ equal to 10 , 5,1 , and $1 / 5$, respectively. With the increase in the normalized vortex scale $L / \rho_{i}$ from 1 to 3 , the vortex velocity varies from $\bar{u}=1$ to $\bar{u}_{\max }$, which equals the normalized velocities $3.7,2.8,1.6$, or 1.2 when $\mu_{*}=10,5,1$, or $1 / 5$, respectively.

obtain the relation that connects the vortex scale $L, L=\kappa^{-1}$, with vortex velocity $u$ normalized to the ion diamagnetic velocity, $\bar{u} \equiv u / v_{i D}$,

$$
\frac{L}{\rho_{i}}=\left[\frac{\bar{u} \mu_{*}\left(1+T_{e} / T_{i}\right)}{\left(\bar{u}+T_{e} / T_{i}\right)\left(\mu_{*}+\bar{u}-\bar{u}^{2}\right)}\right]^{1 / 2} .
$$

Figure 1 shows the dependence of the normalized vortex spatial scale $L / \rho_{i}$ as a function of the normalized vortex velocity $\bar{u}$. We note that the waves with finite $\mu_{*}$ correspond to the drift-Alfvén waves, whereas in the limiting cases $\mu_{*} \rightarrow \infty$ or $\mu_{*} \rightarrow 0$, they correspond to the kinetic Alfvén $\left(L_{n} \rightarrow \infty\right)$ or ion-drift $(\alpha \rightarrow 0)$ waves, respectively. The vortex structures propagating perpendicular to the ambient magnetic field with velocities smaller than the diamagnetic ion drift velocity, $u \leqslant v_{i D}$, possess spatial scales smaller than the ion Larmor radius, $L \leqslant \rho_{i}$ and vice versa. Equation (25) shows that in the homogeneous plasma, $v_{i, e D} \rightarrow 0$, the kinetic Alfvén wave vortices exist when their scale exceeds the ion Larmor radius, $L \geqslant \rho_{i}$.

Let us now analyze the vortex impedance. The latter is given by

$$
\frac{E_{\perp}}{B_{\perp}}=v_{A} D,
$$

where

$$
D=\frac{1}{\mu_{*}^{1 / 2} \bar{u}} \frac{\bar{u}^{2}+\left(T_{e} / T_{i}\right)\left(\mu_{*}+\bar{u}\right)}{1+T_{e} / T_{i}} .
$$

In the cold electron temperature limit, the vortex impedance scales as $D=\bar{u} / \mu_{*}^{1 / 2}$ and thus increases with the growth in $\bar{u}$. However, the incorporation of the finite electron temperature 


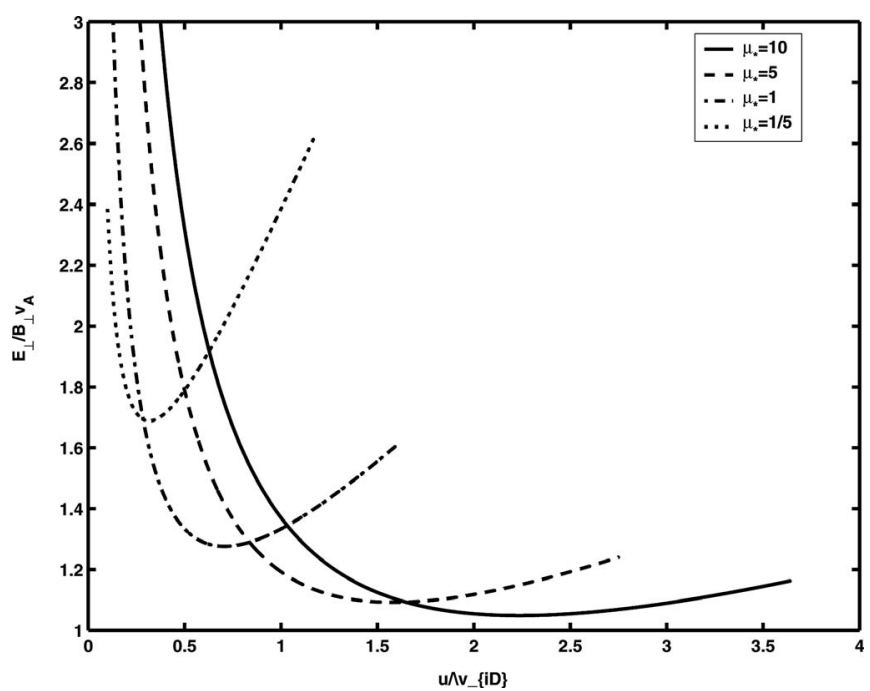

FIG. 2. Same as in Fig. 1 but for the normalized vortex impedance. The vortices propagating with the normalized velocities $\bar{u}$ equal to $2.2,1.6,0.7$, or 0.3 have minimum of the normalized impedance $1,1.1,1.3$, or 1.7 when $\mu_{*}=10,5,1$, or $1 / 5$, respectively.

effects results in the appearance of the minimum in variation of $D$ versus $\bar{u}$. This value is attained at $\bar{u}=\bar{u}^{*}$ $\equiv\left(\mu_{*} T_{e} / T_{i}\right)^{1 / 2}$ and the smallest value of the impedance is $v_{A} D_{\min }$ with

$$
D_{\min }=\frac{\left(T_{e} / T_{i}\right)^{1 / 2}}{1+T_{e} / T_{i}}\left[2+\left(\frac{T_{e} / T_{i}}{\mu_{*}}\right)^{1 / 2}\right] .
$$

The variation of the normalized vortex impedance versus $\bar{u}$ is depicted in Fig. 2. Figures 1 and 2 show that the small-scale vortices with $L<\rho_{i}$ and large impedance, $E_{\perp} / B_{\perp} \gg v_{A}$, propagate with small velocities $\bar{u} \ll 1$. We note that the vortex structures of the drift-Alfvén waves with large spatial scales $L \gg \rho_{i}$ propagating with velocities close to $u \simeq u_{\max }$ have been investigated in Refs. 6-14.

\section{SUMMARY}

Our analysis represents an extension of the previous study of nonlinear drift-Alfvén waves, ${ }^{1,6,7,11,12}$ which was limited to consideration of waves with the spatial scales $L$ larger than the ion Larmor radius $\rho_{i}$. We have extended the previous analyses to the case of the arbitrary ratio $L / \rho_{i}$ using the so-called Padé approximation. Particular attention has been paid to the waves with spatial scales of the order of the ion Larmor radius. In the quasistationary regime, a set of equations describing the nonlinear dynamics of the driftAlfvén waves has been reduced to a single equation that possesses a solution in the form of the dipolar vortex. The basic results of our analysis can be summarized as follows:

(i) It has been shown that the velocity $u$ of the solitary drift-Alfvén vortex structures is localized in the range $0 \leqslant u \leqslant u_{\max }$.

(ii) The variation of the vortex scale $L$ versus vortex velocity $u$ has been analyzed, and the corresponding results have been depicted in Fig. 1. It has been found that the vortex structures propagating faster than the diamagnetic ion velocity, $u \geqslant v_{i D}$, possess spatial scales greater than the ion Larmor radius, $L \geqslant \rho_{i}$, and vice versa.

(iii) The variation of the vortex impedance as the function of the vortex velocity $u$, shown in Fig. 2, has been investigated. It has been found that when the vortex speed is close to the electron drift velocity, the driftAlfvén and the electron-drift modes are coupled. Due to this coupling, the vortex impedance can have a minimum value that is attained when $\bar{u}=\bar{u}^{*}$ $\equiv\left(\mu_{*} T_{e} / T_{i}\right)^{1 / 2}$. The existence of the minimum in the vortex impedance provides the most favorable conditions for the generation of the drift-Alfvén vortex structures.

The Cluster observations reveal the existence of smallscale drift-Alfvén vortex structures with the impedance of the order of the Alfvén speed and characteristic spatial scales $L \simeq(1-3) \rho_{i}$. The theoretical results obtained allow us to modify the existing interpretation of the vortex structures in the cusp region. Figures 1 and 2 show that when $\mu_{*} \simeq 5$ -10 , the vortex structures possess the smallest impedance $\simeq(1-1.1) v_{A}$, they propagate with velocities $u \simeq(1.6$ $-2.2) v_{i D}$, and they have the spatial scales $L \simeq(1.2-1.3) \rho_{i}$. The existence of the structures at the ion Larmor radius scale in the cusp can be explained as the result of their preferable generation in the vicinity of the vortex impedance minimum. Our theoretical results are in reasonable agreement with the Cluster observations of the drift-Alfvén vortices. ${ }^{3,4}$

\section{ACKNOWLEDGMENTS}

This research was supported by the CNRS-Russian Academy collaboration through project No. 19296, by PPARC through Grant No. PP/D002087/1, the Russian Fund for Basic Research (Grant Nos. 07-05-00774, 06-05-65176, and 08-05-00617), by the Program of the Russian Academy of Sciences No. 16, "Solar activity and physical processes in the Solar-Earth system," and by ISTC through Grant No. 3520 .

${ }^{1}$ V. M. Chmyrev, S. V. Bilichenko, O. A. Pokhotelov, V. A. Marchenko, V. I. Lazarev, A. V. Streltsov, and L. Stenflo, Phys. Scr. 38, 841 (1988).

${ }^{2}$ M. L. Goldstein, Nature (London) 436, 782 (2005).

${ }^{3}$ D. Sundkvist, V. Krasnoselskikh, P. K. Shukla, A. Vaivads, M. Andre, S. Buchert, and H. Reme, Nature (London) 436, 825 (2005).

${ }^{4}$ O. Alexandrova, A. Mangeney, M. Maksimovich, N. Cornilleau-Wehrlin, J.-M. Bosqued, and M. Andre, J. Geophys. Res. 111, A12208, DOI: 10.1029/2006JA011934 (2006).

${ }^{5}$ A. B. Mikhailovskii, S. V. Novakovskii, and O. G. Onishchenko, Sov. Phys. JETP 67, 1386 (1988).

${ }^{6}$ V. I. Petviashvili and O. A. Pokhotelov, JETP Lett. 42, 54 (1985).

${ }^{7}$ J. Liu and W. Horton, J. Plasma Phys. 36, 1 (1986).

${ }^{8}$ P. K. Shukla, M. Y. Yu, and L. Stenflo, Phys. Rev. A 34, 3478 (1986).

${ }^{9}$ V. P. Lakhin, A. B. Mikhailovskii, and O. G. Onishchenko, Sov. J. Plasma Phys. 13, 188 (1987).

${ }^{10}$ V. I. Petviashvili and O. A. Pokhotelov, Solitary Waves in Plasmas and in the Atmosphere (Gordon and Breach, Langhorne, 1992).

${ }^{11}$ V. P. Lakhin, S. V. Makurin, A. B. Mikhailovskii, and O. G. Onishchenko, J. Plasma Phys. 38, 407 (1987).

${ }^{12}$ A. B. Mikhailovskii, V. P. Lakhin, G. D. Aburdzaniya, L. A. Mikhailovskaya, O. G. Onishchenko, and A. I. Smolyakov, Plasma Phys. Controlled Fusion 29, 1 (1987). 
${ }^{13}$ A. B. Mikhailovskii and O. G. Onishchenko, Plasma Phys. Rep. 21, 357 (1995).

${ }^{14}$ O. A. Pokhotelov, L. Stenflo, and P. K. Shukla, Plasma Phys. Rep. 22, 852 (1996).

${ }^{15}$ A. B. Mikhailovskii, Electromagnetic Instabilities in an Inhomogeneous Plasma (IOP, Bristol, 1992).

${ }^{16}$ B. N. Kuvshinov and A. B. Mikhailovskii, Plasma Phys. Rep. 22, 529 (1996).

${ }^{17}$ C. C. Chaston, T. D. Phan, J. W. Bonnell et al., Phys. Rev. Lett. 95, $065002(2005)$.
${ }^{18}$ J. R. Johnson and C. Z. Cheng, Geophys. Res. Lett. 24, 1423, DOI: 10.1029/97GL01333 (1997).

${ }^{19}$ A. V. Streltsov, W. Lotko, J. R. Johnson, and C. Z. Cheng, J. Geophys. Res. 103, 26559, DOI: 10.1029/98JA02679 (1998).

${ }^{20}$ A. Hasegawa and C. Uberoi, The Alfvén Wave (U.S. Department of Energy, Washington, D.C., 1982).

${ }^{21}$ O. G. Onishchenko, O. A. Pokhotelov, V. P. Pavlenko, P. K. Shukla, T. Farid, L. Stenflo, F. F. Kamenets, and A. V. Bogdanov, Phys. Plasmas 8, 59 (2001). 